\title{
KN11 | Enhancing The Success Of Macromolecular Crystallization
}

Chayen, Naomi (Imperial College London, London, GBR)

Obtaining high quality crystals is a pivotal step to facilitate the structure determination of proteins and other biological macromolecules by X-ray crystallography.

In spite of momentous progress in the miniaturisation, automation and analysis of crystallisation experiments, the production of useful crystals still presents a major barrier to structure determination [1,2].

There is no 'magic bullet' that will guarantee well-diffracting crystals, hence rational approaches leading to the development of new and improved technologies for obtaining high quality crystals is of crucial importance to progress.

This talk will present strategies for increasing the chances of success and highlight a variety of practical methods that resulted in successful crystallization when standard procedures had failed [3-7]. The methods involve active influence and control of the crystallization environment in order to lead crystal growth to the desired result. Many of the techniques are automated for adaptation to high throughput mode and several have been patented and commercialised.

[1] Chayen and Saridakis (2008) Nature Methods 5, 147-153.

[2] Chayen, Helliwell and Snell Macromolecular Crystallization and Crystal Perfection, Oxford Univ. Press, Oxford, UK (2010).

[3] Saridakis et al. (2011) Proc. Natl. Acad. Sci. U.S.A. 108, 11081-11086.

[4] Khurshid et al. (2014) Nature Protocols 9,Pages: 1621-1633.

[5] Govada et al. (2016) Scientific Reports Nature Publishing Group 6:20053.

[6] Nanev et al. (2017) Scientific Reports Nature Publishing Group 7:35821.

[7] Govada and Chayen (2019) Crystals 9(2), 106. 